\title{
Gastroenterologie
}

\section{Lactobacillus reuteri bei Säuglingskoliken}

\section{Eine aktuelle Metaanalyse kam zu interessanten Ergebnissen.}

Säuglingskoliken sind bei Kindern unter drei Monaten sehr verbreitet und stellen für die ganze Familie eine belastende Situation dar. Die Symptome werden meist mit der modifizierten Wessel'schen Skala, die Kriterien wie Weinen und/oder Unruhe als Maß für die Belastung einbezieht, erfasst. Lacotbacillus reuteri DSM 17938 hat sich in zahlreichen Studien als vielversprechende Behandlungsmethode bei Säuglingskoliken erwiesen; ein Konsensus hinsichtlich ihrer Wirksamkeit konnte allerdings bislang nicht erzielt werden.

Die Forscher um Valerie Sung versuchte daher, durch eine MetaAnalyse der vorliegenden Daten, herauszufinden, ob die Gabe dieses Probiotikums die Parameter Weinen und Unruhe bei Säuglingen mit Koliken beeinflusst und ob etwaige Effekte von der Fütterungsmethode abhängig seien.

\section{Datenquellen}

Die Forschergruppe zog Online Daten (PubMed, Embase, the Cumulative Index to Nursing and Allied Health Literature, the Database of Abstracts of Reviews of Effects, and Cochrane), e-Abstracts und die Register klinischer Studien als Quellen heran. Selektiert wurden doppelblinde randomisierte, kontrollierte Studien, in welchen L. reuteri DSM 17398 vs. Placebo oral an Säuglinge, die an Koliken litten, verabreicht wurde. Verzeichnet wurde die Dauer des Weinens oder der Unruhe als Maß für den Behandlungserfolg über 21 Tage.

Die Rohdaten der Studienteilnehmer generierten die Forscher aus den inkludierten Studien, die gleichzeitig in Form von Regressionsmodellen dargestellt wurden. Eine detaillierte Beschreibung der Methode wurde im Studienprotokoll niedergelegt.

\section{Studienauswahl}

Vier doppelblinde internationale Studien, die insgesamt 345 Säuglinge mit Koliken (174 probiotisch behandelte, 171 Placeboempfänger) inkludierten, wurden in die Metaanalyse eingeschlossen. Die Probiotikagruppe war dadurch gekennzeichnet, dass die Säuglinge weniger weinten und/ oder unruhig waren als die Probanden der Placebogruppe, und zwar zu allen Zeitpunkten (Tag 21, adjustierte mittlere Differenz - Veränderungen der Ausgansgwerte in Minuten: -25.4 (95\% confidence interval (Cl); -47.3 bis -3.5$)$. Die probiotische Gruppe kam fast zweimal so häufig in den Genuss, einen Therapieerfolg zu erleben, als die Placebogruppe, zu allen Zeitpunkten der vorliegenden Studie (Tag 21, adjustierte incidence ratio (IR) 1.7 (95\% Cl: 1.4 bis 2.2.). Signifikant ausgeprägte Wirkungen der Probiotikagabe zeichneten sich bei gestillten Säuglingen ab (number needed to treat für Tag 21 Erfolg: 2.6 (95\% Cl: 2.0 bis 3.6)), nicht signifikante allerdings bei Formulaernährten Säuglingen.

\section{Ergebnisse}

Lactobacillus reuteri DSM 17938 ist wirksam und kann bei gestillten Säuglingen, die an einer Kolik leiden, zur Milderung der Beschwerden verabreicht werden. Seine Rolle und Einsatzmöglichkeiten bei Formula-ernährten Säuglingen, die an Koliken leiden, sollte Gegenstand weiterer Studien sein.

Paediatr. Paedolog. Austria 2018 · 53:91 https://doi.org/10.1007/s00608018-0561-x

(c) Springer-Verlag GmbH Austria, ein Teil von Springer Nature 2018
Quelle: Pediatrics. 2018 Jan;141(1). pii: e20171811. https:// doi.org/10.1542/ peds.2017-1811. Lactobacillus reuteri to Treat Infant Colic: A Meta-analysis. Sung V, D'Amico F, Cabana MD, Chau K, Koren G, Savino F, Szajewska H, Deshpande G, Dupont C, Indrio F, Mentula S, Partty A, Tancredi D. 